\title{
Seed Ageing and Meiotic Chromosomal Abnormalities in Lettuce
}

\author{
N. K. Rao' and E. H. Roberts \\ Department of Agriculture, University of Reading, \\ Earley Gate, P. O. Box 236, Reading RG6 2AT, UK
}

Accepted July 30, 1988

In a recent study on stored lettuce (Lactuca sativa L.) seeds we showed that loss of seed viability is associated with an accumulation of chromosome damage in the surviving seeds (see Rao et al. 1987). Like many other previous investigators we evaluated the chromosome damage in the primary roots by examining the anaphase cells of the first mitotic divisions where the chromosomes containing lesions induced during storage form visible aberration configurations.

Aberrant cells containing gross chromosome damage were however shown to be removed subsequently by diplontic selection since they occasion mechanical separation problems during divisions and produce daughter cells which are genetically imbalanced. Structural changes in the chromosomes involving rearrangement of segments such as translocations and inversions, however, do not form aberrant configurations in mitotic cells, hence they are not subjected to selection, and survive through successive cell generations to become visible only at meiosis. As is well known, translocations and inversion heterozygotes produce gametes containing duplications and deficiencies which, as a result are inviable and cause considerable sterility in the plants carrying them. Also small deficiencies may escape diplontic selection and survive up to meiosis and induce sterility since, being haploid, the gametophyte is extremely sensitive to even small genetic losses. Therefore a critical examination of the meiotic divisions is necessary to expose these important changes in the genome that might have been induced during seed storage. There are only a few previous instances where the meiotic chromosomal aberrations have been investigated in mature plants grown from aged seeds (see Murata et al. 1984). In this paper we report the effect of seed ageing on meiosis and on the pollen and seed fertility in lettuce.

\section{Materials and methods}

Plants grown from lettuce seeds (achenes) aged under the storage conditions listed in Table 1 were used in this study. Fifteen to twenty plants from each of the storage treatments were grown in plastic pots containing Levington Universal compost in a glasshouse under long-day conditions. Meiosis was studied in the pollen mother cells (PMCs) in three randomly selected inflorescences per plant using three flower buds from each inflorescence. The inflorescences were fixed in Carnoy's fixative (6:3:1 mixture of ethanol, chloroform and acetic acid) for 24 hours, washed in distilled water and stored in $70 \%$ ethanol until squashes were made. All samples grown in a single year were fixed on the same day, as far as possible. The flower buds were dissected and the anthers were squashed in $1 \%$ acetocarmine solution. The first metaphase (MI), first and second anaphase (AI and AII) stages of the meiotic divisions were examined for any chromosomal abnormalities. Approximately, 500 PMCs were scored at each of the three meiotic stages in each treatment.

Pollen fertility was determined on the basis of stainability with $1 \%$ acetocarmine using anthers from two mature but unopened florets, each from three different inflorescences random-

${ }^{1}$ Present address: Genetic Resources Unit, International Crops Research Institute for the Semi-Arid Tropics (ICRISAT), Patancheru P. O., Andhra Pradesh 502 324, India. 
ly selected on each plant. A minimum of 500 pollen grains were scored per plant and pollen fertility was expressed as the percentage of dark stained grains of the total number of grains observed.

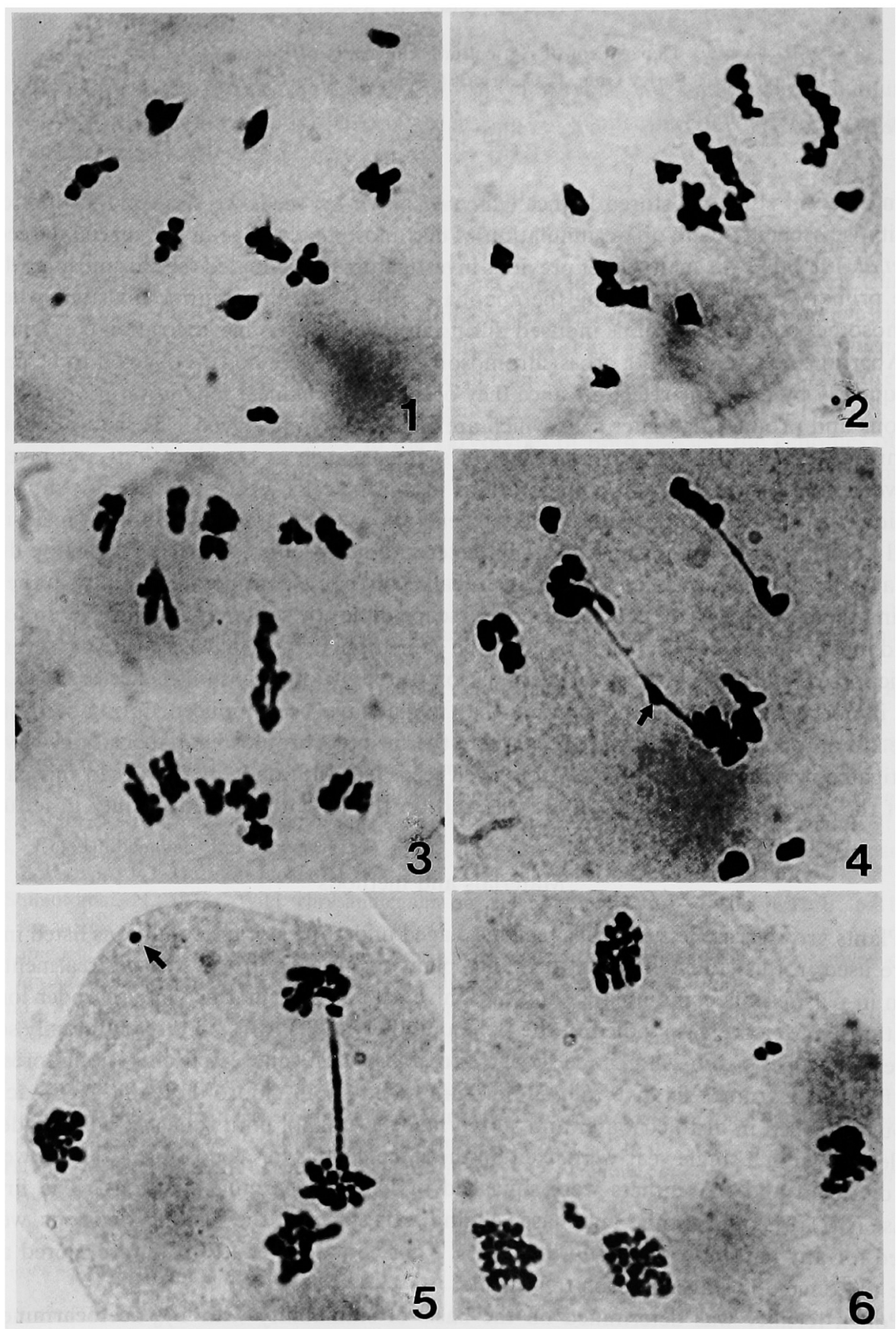

Figs. 1-6. Meiotic chromosomal aberrations in lettuce. All figs. $\times 1120$. 1 , metaphase I: 8 bivalents and 2 univalents. 2, metaphase $I$ : precocious segregation. 3, anaphase I: late disjunction. 4, anaphase I: two bridges and one acentric fragment $(\rightarrow)$. 5, anaphase II: one bridge and one acentric fragment $\Leftrightarrow$, 6 , anaphase II: laggards. 


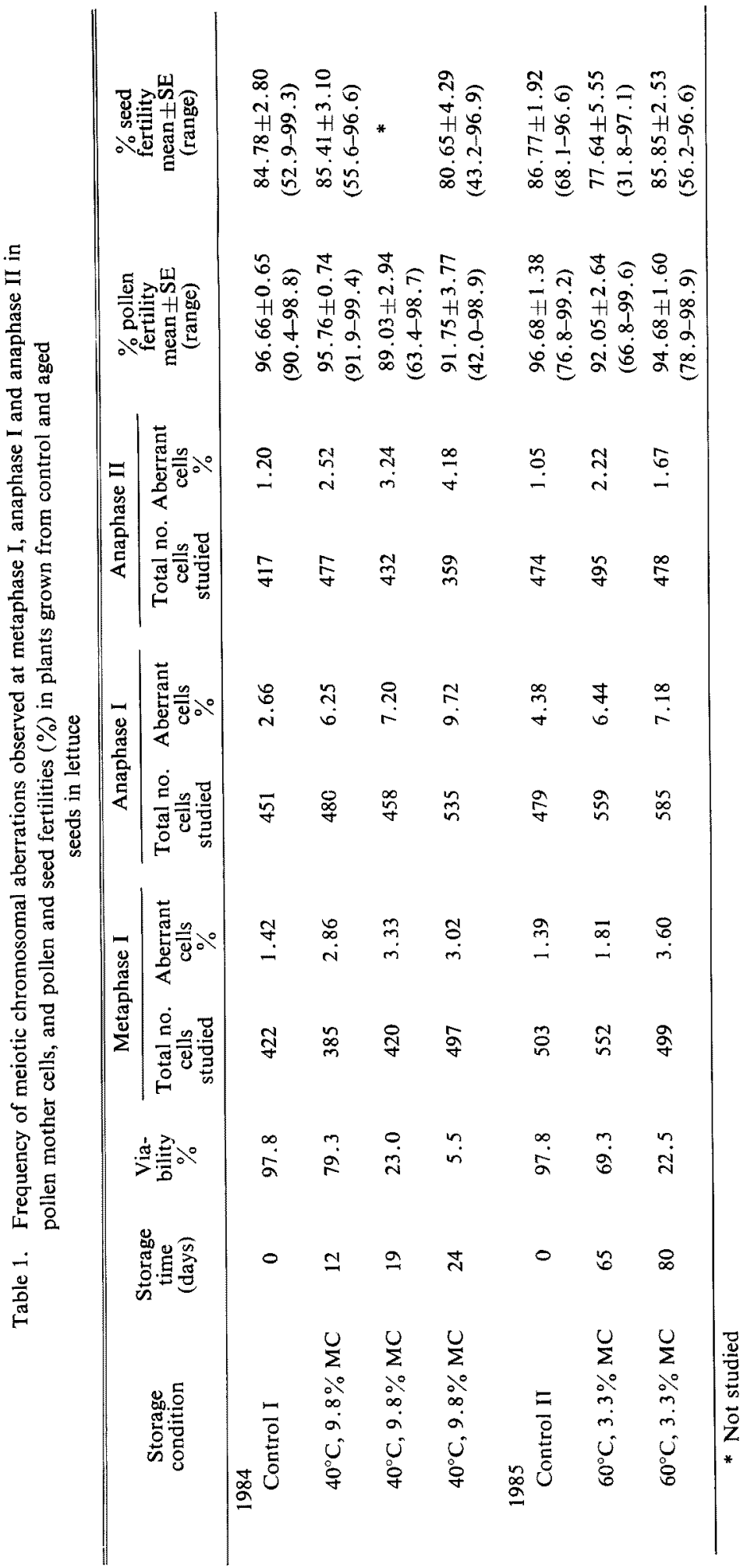


Seed fertility was determined from ten fruiting heads randomly selected from each mature plant and expressed as the percentage of full achenes of the total number of achenes.

\section{Results and discussion}

\section{Meiotic chromosomal abnormalities}

The experiment extended over two seasons, hence a separate control (from the original seed stock of 97.8 per cent viability which had not been deliberately aged) was grown to compare with the ageing treatments grown in the same year. Chromosomal abnormalities were found in all stages of meiosis in plants grown from the control as well as the aged seed lots. The various types of abnormalities observed in meiosis are shown in Figs. 1-6, and the percentage of PMCs showing abnormal divisions in the various stages of meiosis are given in Table 1.

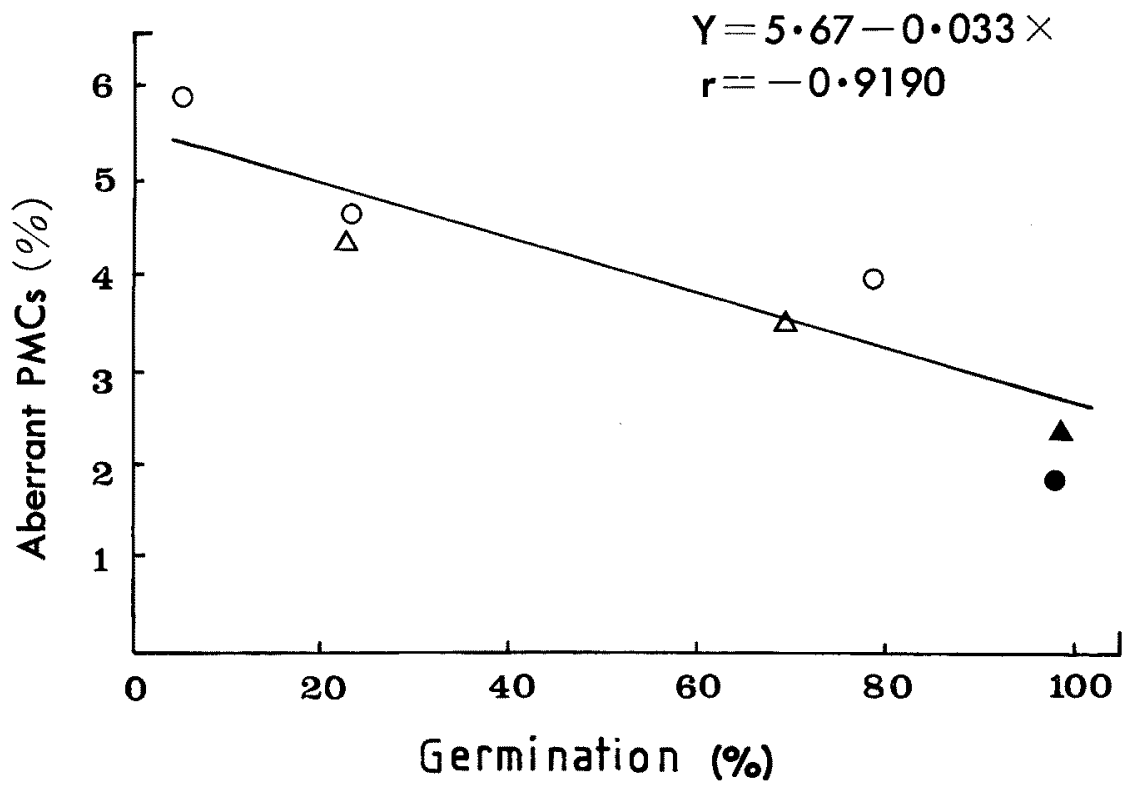

Fig. 7. The relationship between seed viability and the frequency of aberrant pollen mother cells (PMCs) at metaphase I, anaphase I and anaphase II stages of meiosis in plants grown from aged lettuce seeds. (C) control I; ( $\mathbf{A}$ ) control II; seeds aged at $(\mathrm{O}) 40^{\circ} \mathrm{C}$ and $9.8 \%$ moisture content and $(\triangle) 60^{\circ} \mathrm{C}$ and $3.3 \%$ moisture content.

In Fig. 7 the total frequency of aberrant cells observed in the three stages of meiosis is plotted against seed viability (percentage normal germination). As can be seen, there is an increase in aberrant cells with loss of seed viability, and although the significance is not known, there appears to be a common linear relationship between the two parameters regardless of the storage condition in which the seed had been initially aged.

The types and proportion of aberrations observed at each of the three stages of meiosis are summerised in Table 2. Among the abnormalities observed in metaphase I, PMCs with two univalents occurred relatively frequently, followed by cells with acentric fragments. Precocious segregation was also observed in a few cases. Associations of two or more bivalents resembling the configurations of a translocation heterozygote were seen in a few plants grown from aged seeds, however later stages of meiosis were normal in those cases. These associations are presumably due to the stickiness of the chromosomes induced probably as a consequence of 
seed ageing.

Among the abnormalities observed in anaphase I, structures similar to inversion bridges; with or without associated fragments, were observed most frequently. Lagging chromosomes and fragments which appeared to have resulted from broken bridges during chromosome separation were observed fairly commonly in late anaphase cells.

The presence of anaphase bridges associated with fragments is generally an indication of inversion heterozygosity. However, Lindqvist (1960) considered that inversions occur rarely in pure lines and the frequency of bridges observed in some forms of lettuce was far too high to be accounted in this way. Lewis and John (1966) also questioned the evidence presented in several earlier investigations suggesting the occurrence of inversions in a number of species and emphasised the need to exercise caution in the interpretation of anaphase bridges at meiosis. They showed that dicentric bridges with associated fragments could result from iso-locus breakage of sister chromatids followed by sister reunion, and such breakages could be the out

Table 2. Types and frequencies of chromosomal aberrations observed at metaphase I (MI), anaphase I (AI) and anaphase II (AII) stages of meiosis in plants grown from aged lettuce seeds

\begin{tabular}{lc}
\hline $\begin{array}{c}\text { Stages of meiosis and type of } \\
\text { of aberrations }\end{array}$ & $\begin{array}{c}\text { Number and proportion (\%) } \\
\text { of aberrant cells }\end{array}$ \\
\hline Metaphase I & $46(67.65)$ \\
Univalents & $15(22.06)$ \\
Fragments & $7(10.29)$ \\
Precocious separation & $68(100.00)$ \\
Total no. of aberrant cells & 2353 \\
Total no. of MI cells studied & $111(57.51)$ \\
Anaphase I & $62(32.12)$ \\
Bridges with or without fragments & $20(10.36)$ \\
Fragments & $193(99.99)$ \\
Laggards & 2617 \\
Total no. of aberrant cells & \\
Total no. of AI cells studied & $18(30.00)$ \\
Anaphase II & $32(53.33)$ \\
Bridges with or without fragments & $10(16.67)$ \\
Fragments & $60(100.00)$ \\
Laggards & 2241 \\
Total no. of aberrant cells &
\end{tabular}

come of errors in the mechanism of chiasma formation. In the present studies, since the critical pairing configurations in the pachytene stage have not been studied, it is difficult to conclude unequivocally that the observed anaphase bridges in anaphase I owe their origin to inversion heterozygosity. Whatever the causal mechanism was, bridges were observed with increased frequency in populations grown from aged seeds.

There was some decrease in the frequency of aberrant cells in anaphase II compared to anaphase I; nevertheless a general increase in the frequency of aberrant cells in anaphase II was noticed in the plants grown from aged seeds compared to those from the control seeds (Table 1). Among the meiotic aberrations observed in anaphase II, fragments were relatively common, followed by bridges with or without fragments and lagging chromatids.

\section{Pollen and seed fertility}

The data on pollen and seed fertility are presented in Table 1. Although the mean pollen 
and seed fertilities did not differ significantly from the controls, there was an increase in the spread of distribution for pollen and seed sterility in the populations from aged seeds. Probably an insufficient number of plants were used in the present investigations to expose any differences that there might have been. As expected pollen and seed sterility was high in the plants showing a large number of abnormalities in meiosis. However, such a correlation did not always exist as plants with apparently normal meiosis sometimes exhibited a high degree of sterility. This could easily have been due to sampling error since only three heads per plant were studied and the degree of irregularity observed in meiotic abnormalities varied greatly within the same plant from head to head, and possibly from flower to flower within the same inflorescence. Also, the deleterious role of invisible and therefore undetected deficiencies in meiosis, and the unmasking of lethal gene mutations in the haploid state of the gametophyte in inducing sterility cannot be discounted.

Meiotic chromosomal aberrations in plants grown from aged seeds have been investigated by only a few in the past. Translocations, deficiencies and other meiotic disturbances were reported in plants grown from aged Datura seeds by Cartledge and Blakeslee (1934), Cartledge et al. (1936) and Avery and Blakeslee $(1936,1943)$. Gunthardt et al. (1953) reported chromosomal interchanges in the PMCs of plants grown from 2-3 year old hexaploid wheat and 32 year old barley and tetraploid wheat seeds. Murata et al. (1984), however, although observing bridges and fragments in anaphase and telophase stages of meiosis in plants grown from artificially aged seeds of barley, found no clear differences when compared with controls.

Reduced pollen fertility, assumed to have been caused by chromosomal and gene mutations, was reported in plants grown from aged seeds of Datura (Cartledge and Blakeslee 1934 and Cartledge et al. 1936), wheat (Shkvarnikov 1937), tobacco (Gisquet et al. 1951), perennial ryegrass (Griffiths and Pegler 1964), barley, broad bean and pea (Abdalla and Roberts 1969) and soybean and barley (Chauhan and Swaminathan 1984). In contrast to all these reports, Murati et al. (1984) found no significant differences in pollen and seed fertilities between the control and ageing treatments in barley.

\section{Summary}

Meiotic chromosomal abnormalities observed in lettuce plants grown from aged seeds increased with the decrease in the viability of the seed lot. Univalents, fragments and a few cases of precocious segregation were observed in metaphase I. Abnormalities found at anaphase I and anaphase II included dicentric bridges with or without fragments, acentric fragments and lagging chromosomes and chromatids.

The spread of the distribution for pollen and seed sterility increased considerably in some ageing treatments; however, average pollen and seed fertilities were not significantly different from the control.

\section{Acknowledgements}

We are most grateful to the Association of Commonwealth Universities for providing financial support and to ICRISAT for granting study leave to N.K.R. which enabled this work to be undertaken.

\section{References}

Abdalla, F. H. and Roberts, E. H. 1969. The effects of temperature and moisture on the induction of genetic changes in seeds of barley, broad beans and peas during storage. Ann. Bot. 33: 153-167. 
Avery, A. G. and Blakeslee, A. F. 1936. Visible mutation from aged seeds. Am. Nat. 70: 36-37.

- and - 1943. Mutation rate in Datura seed which had been burried 39 years. Genetics 28: 69-70.

Cartledge, J. L. and Blakeslee, A. F. 1934. Mutation rate increased by aging seeds as shown by pollen abortion. Proc. Natl. Acad. Sci. 20 : $103-110$.

-, Barton, L. V. and Blakeslee, A. F. 1936. Heat and moisture as factors in the increased mutation rate from Datura seeds. Proc. Am. Phil. Soc. 76: 663-685.

Chauhan, K.P.S. and Swaminathan, M. S. 1984. Cytogenetical effects of ageing in seeds. Genetica 64: 6976.

Gisquet, P., Hitier, H., Izard, C. and Mounat, A. 1951. Mutations naturelles observées chez N. tabacum L. et mutations expérimentales provoquées par l'extrait à froid de graines vieilles prématurement. Ann. Inst. Exp. Tabac. Bergerac 1: 5-36.

Griffiths, D. J. and Pegler, R. A. D. 1964. The affects of long-term storage on the viability of S23 perennial ryegrass seed and on subsequent plant development. J. Br. Grassl. Soc. 19: 183-190.

Gunthardt, H., Smith, L., Haferkamp, M. E. and Nilan, R. A. 1953. Studies on aged seeds. 2. Relation of age of seeds to cytogenetic effects. Agron. J. 45: 438-441.

Lewis, K. R. and John, B. 1966. The meiotic consequences of spontaneous chromosome breakage. Chromosoma 18 : 287-304.

Lindqvist, K. 1960. Cytogenetic studies in Serriola group of Lactuca. Hereditas 46: $75-151$.

Murata, M., Tsuchiya, T. and Roos, E. E. 1984. Chromosome damage induced by artificial seed aging in barley. 3. Behavior of chromosomal aberrations during plant growth. Theor. Appl. Genet. 67: 161-170.

Rao, N. K., Roberts, E. H. and Ellis, R. H. 1987. Loss of viability in lettuce seeds and the accumulation of chromosome damage under different conditions. Ann. Bot. 60: 85-96.

Shkvarnikov, P. 1937. Über Erhöhung der Mutationsräte bei Weizen nach langer Aufbetwahrung der Samen. Genetica 19: 188-199. 